\title{
CHAIN CONDITIONS ON POSETS
}

\author{
by CYNTHIA D. GEOFFROY $\dagger$ and H. E. SCHEIBLICH
}

(Received 18 December, 1972; revised 10 July, 1973)

1. Introduction and definitions. The aim of this note is to generalize to an arbitrary partially ordered set (poset) $(P, \leqq)$ the standard lattice results on the Jordan-Dedekind Chain Condition (abbreviated hereafter to J.D.C.C.). Birkhoff [1] defines semimodularity for a lattice $L$ by

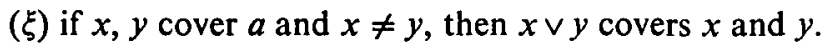

The additional assumption that $L$ is of finite length is heavily relied upon in proving that the J.D.C.C. holds [1, Theorem 3, p. 68].

The semimodularity condition $(\xi)$ has a natural generalization to an arbitrary poset $P$ by

$(\sigma)$ if $x, y$ cover $a$ and $x \neq y$, then there exists a $d \in P$ which covers $x$ and $y$.

For a lattice the conditions $(\xi)$ and $(\sigma)$ coincide and the following is true. If $P$ is a semimodular poset of finite length, then the J.D.C.C. holds.

In [2], Rhodes has given a definition of semimodularity for a lower semilattice $S$. His result is that, if $S$ satisfies a strong semimodularity condition, then $S$ satisfies a strong chain condition.

Let $(P, \leqq)$ be a poset, and let $a, b \in P$. Then $b$ covers $a(b>a, a \prec b)$ if and only if $a<b$ and $\{x \in P: a \leqq x \leqq b\}=\{a, b\}$. Also, if $x, y \in P$, then $x \wedge y$ and $x \vee y$ mean, respectively, the greatest lower bound and least upper bound of $\{x, y\}$ if they exist. Thus $x \wedge y=a$ means that $x \wedge y$ exists and equals $a$. A similar statement holds for $x \vee y$.

Definition 1.1. Let $P$ be a poset. Then $P$ is called

(i) strongly upper semimodular if and only if, whenever $a \wedge b, a \vee b$ exist and $a>a \wedge b$, then $a \vee b>b$;

(ii) weakly upper semimodular if and only if, whenever $a \wedge b$ exists and $a, b \succ a \wedge b$, then $a \vee b$ exists and $a \vee b>a, b$.

Definition 1.2. Let $P$ be a poset. Then $P$ satisfies

(i) the strong chain condition if and only if, whenever $a<b$ and there is a finite maximal chain from $a$ to $b$, then all maximal chains from $a$ to $b$ are finite and have the same length;

(Note. The Axiom of Choice implies that, if this condition is satisfied, then every chain from $a$ to $b$ is finite.)

(ii) the weak chain condition if and only if, whenever $a<b$ and there is a finite maximal chain from $a$ to $b$, all finite maximal chains from $a$ to $b$ have the same length.

It will be shown that, if $\boldsymbol{P}$ is a poset which is strongly (weakly) upper semimodular, then $\boldsymbol{P}$

† This paper is part of the first author's M.S. thesis, written at the University of South Carolina under the direction of the second author. 
satisfies the strong (weak) chain condition. An example will show that the semimodularity conditions and chain conditions are not the same, even in a lattice. A further example will show that the weak semimodularity condition cannot be further relaxed and still imply the weak chain condition.

2. The chain conditions. Let $P$ be a partially ordered set and let $a, x, b \in P$ with $a<x<b$. The reader is asked to use Zorn's Lemma to show that there is a chain $C$ from $a$ to $b$ which contains $x$ and which is maximal in the collection of all chains from $a$ to $b$.

Throughout, $\mathbb{N}$ will denote the set of positive integers. Whenever $C$ is a finite chain in a poset $P$, then $L(C)$ will denote the length of $C$.

THEOREM 2.1. Let $P$ be a strongly upper semimodular poset. Then $P$ satisfies the strong chain condition.

Proof. (By induction). Let $K=\left\{n \in \mathbb{N}:\right.$ if $a, b \in P$ with $a<b$ and $C_{1}$ is a finite maximal chain from $a$ to $b$ of length $n$ and $C_{2}$ is a finite chain from $a$ to $b$, then $\left.L\left(C_{2}\right) \leqq n\right\}$. It is enough to show that $K=\mathbb{N}$.

Certainly $1 \in K$. Assume now that $n \in \mathbb{N}, 1<n$, and $t \in K$ whenever $1 \leqq t<n$. Let $a=a_{0} \prec a_{1} \prec \ldots \prec a_{n}=b$ be a maximal chain from $a$ to $b$, and let $C$ be a finite chain from $a$ to $b$.

Case (i). There exist $x \in C-\{a, b\}$ and $t \in[1, n-1]$ such that $a_{t} \leqq x$.

If $a_{t}=x$, then, since $a=a_{0} \prec a_{1} \prec \ldots \prec a_{t}=x$ and $x=a_{t} \prec a_{t+1} \prec \ldots \prec a_{n}=b$ are maximal chains from $a$ to $x$ and from $x$ to $b$ of lengths $t, n-t \in K$, then

$$
L(C)=L\{y \in C: y \leqq x\}+L\{y \in C: x \leqq y\} \leqq t+(n-t)=n .
$$

Assume now that $a_{t}<x$. Since $a_{t} \prec a_{t+1} \prec \ldots \prec a_{n}=b$ is a maximal chain from $a_{t}$ to $b$ of length $n-t \varepsilon K$, there is a maximal chain from $a_{t}$ to $b$ of length $n-t$ which contains $x$, say $a_{t}=y_{t} \prec y_{t+1} \prec \ldots \prec y_{t+s}=x<y_{t+s+1} \prec \ldots \prec y_{n}=b$. Now, since $a=a_{0} \prec a_{1} \prec \ldots \prec a_{t}=$ $y_{t} \prec y_{t+1} \prec \ldots \prec y_{t+s}=x$ and $x=y_{t+s} \prec y_{t+s+1} \prec \ldots \prec y_{n}=b$ are maximal of lengths $t+s, n-(t+s) \in K$, then

$$
L(C)=L\{y \in C: y \leqq x\}+L\{y \in C: x \leqq y\} \leqq(t+s)+[n-(t+s)]=n .
$$

Case (ii). For each $x \in C-\{a, b\}$ and for each $t \in[1, n-1], a_{t} \ddagger x$. Then, for each $x \in C-\{a, b\}, a_{1} \pm x$ and so $a_{1} \wedge x=a_{0}$.

(iia). For each $x \in C-\{a, b\}, a_{1} \vee x=b$. Then, since $P$ is strongly upper semimodular, $x \prec b$ for each $x \in C-\{a, b\}$. It follows that $L(C) \leqq 2 \leqq n$.

(iib). For each $x \in C-\{a, b\}$, either $x \vee a_{1}$ does not exist or $x \vee a_{1}$ exists but is not equal to $b$. In either case choose $u \in P$ such that $x, a_{1}<u<b$.

Since $a_{1} \prec a_{2} \prec \ldots \prec a_{n}=b$ is maximal from $a_{1}$ to $b$ of length $n-1 \in K$, there is a maximal chain from $a_{1}$ to $b$ of length $n-1$ which contains $u$, say $a_{1}=y_{1} \prec \ldots \prec y_{r}=u \prec y_{r+1} \prec \ldots$ $\prec y_{n}=b$. Since $a=a_{0} \prec a_{1}=y_{1} \prec \ldots \prec y_{r}=u$ is maximal from $a$ to $u$ of length $r \in K$, there is a maximal chain from $a$ to $u$ of length $r$ which contains $x$, say $a=a_{0}=z_{0} \prec z_{1} \prec \ldots \prec z_{s}=$ $x \prec z_{s+1} \prec \ldots \prec z_{r}=u$. Now, since $a=a_{0}=z_{0} \prec z_{1} \prec \ldots \prec z_{s}=x$ and $x=z_{s} \prec z_{s+1} \prec \ldots$ $\prec z_{r}=u \prec y_{r+1} \ldots y_{n}=b$ are maximal of lengths $s, n-s \in K$, it follows that $L(C) \leqq s+(n-s)=$ $n$. 
In any event, $n \in K$ and so $K=\mathbb{N}$.

LemMA 2.2. Let $P$ be a weakly upper semimodular poset. Let $a, b \in P$ with $a<b$ and let $a=a_{0} \prec a_{1} \prec \ldots \prec a_{n}=b$ be a finite maximal chain from $a$ to $b$. Let $x \in P$ be such that $x \leqq a_{i}$ for each $i \in[1, n-1]$ and $a \prec x<b$. Then $x \vee a_{i} \succ x \vee a_{i-1}, a_{i}$ for each $i \in[1, n-1]$.

Proof. (By induction). Let $K=\left\{i \in[1, n-1]: x \vee a_{i} \succ x \vee a_{i-1}, a_{i}\right\}$.

Since $a_{1}, x>a$, it follows that $x \wedge a_{1}=a$. Since $P$ is weakly upper semimodular, then $x \vee a_{1}$ exists and $x \vee a_{1} \succ x, a_{1}$. But $x=x \vee a_{0}$ and so $x \vee a_{1} \succ x \vee a_{0}, a_{1}$. Thus $1 \in K$.

Assume now that $i \in[1, n-2]$ and $i \in K$. Then $x \vee a_{i} \succ a_{i}$ and $a_{i+1} \succ a_{i}$. Notice that $x \vee a_{i} \neq a_{i+1}$ since otherwise $x \leqq a_{i+1}$, contradicting the hypothesis. Since $a_{i+1} \succ a_{i}$, it follows that $\left(x \vee a_{i}\right) \wedge a_{i+1}=a_{i}$. Since $P$ is weakly upper semimodular, then $\left(x \vee a_{i}\right) \vee a_{i+1}>$ $x \vee a_{i}, a_{i+1}$; that is, $x \vee a_{i+1} \succ x \vee a_{i}, a_{i+1}$. Thus $i+1 \in K$.

TheOREM 2.3. Let $P$ be a weakly upper semimodular poset. Then $P$ satisfies the weak chain condition.

Proof. (By induction). Let $K=\{n \in \mathbb{N}:$ if $a, b \in P$ with $a<b$ and there is a finite maximal chain from $a$ to $b$ of length $n$, then all finite maximal chains from $a$ to $b$ have length $n\}$.

Certainly $1 \in K$. Assume now that $n \in K, a<b$, and that $a=a_{0} \prec a_{1} \prec \ldots \prec a_{n+1}=b$, $a=b_{0} \prec b_{1} \prec \ldots \prec b_{m+1}=b$ are finite maximal chains from $a$ to $b$ of lengths $n+1$ and $m+1$, respectively. Consider $b_{1}$ and choose $j$ minimal with respect to $b_{1} \leqq a_{j}$. If $j=1$, then $b_{1}=a_{1}$ since $a_{1}>a$. It follows immediately that $n=m$ and hence $n+1=m+1$. Assume $j \neq 1$. Then $b_{1}<a_{j}$, since $b_{1}>a_{0}$. By Lemma $2.2, b_{1}=b_{1} \vee a_{0} \prec b_{1} \vee a_{1} \prec \ldots \prec b_{1} \vee a_{j-1}=$ $a_{j}<a_{j+1}<\ldots<a_{n+1}=b$ is maximal of length $n$. Thus $n=m$ and again $n+1=m+1$. In any event, $n+1 \in K$ and hence $K=\mathbb{N}$.

EXAmple 2.4. Let $L=\{(x, 0): x \in \mathbb{R}, 0 \leqq x \leqq 1\} \cup\{(1, y): y \in \mathbb{R}, 0 \leqq y \leqq 1\} \cup\{(0,1)\}$, where $\mathbb{R}$ is the set of real numbers. Let $L$ be ordered by the usual cartesian ordering. Then $L$ is a lattice which is weakly upper semimodular, but $L$ does not satisfy the strong chain condition.

Definition 1.1.2 might be considered to be a bit disappointing in the light of Definition 1.1.1. One might hope that 1.1.2 would read that whenever $a \wedge b, a \vee b$ exist and $a, b>a \wedge b$, then $a \vee b>a, b$. The next example is to illustrate that even for a lower semilattice $S$, the weakened definition need not imply the weak chain condition.

EXAMPle 2.5. Let $S=\left\{(0,0),\left(0, \frac{1}{2}\right),\left(\frac{1}{2}, 1\right),\left(\frac{1}{2}, 0\right),(1,0),\left(1, \frac{1}{2}\right),(1,1)\right\} \cup\{(x, x): x \in \mathbb{R}$ and $\left.\frac{1}{2}<x<1\right\}$. Let $S$ be ordered by the usual cartesian ordering. Then $S$ is a lower semilattice such that, whenever $a \wedge b, a \vee b$ exist and $a, b \succ a \wedge b$, then $a \vee b>a, b$. However, there are maximal chains from $(0,0)$ to $(1,1)$ of lengths 3 and 4 .

\section{REFERENCES}

1. Garrett Birkhoff, Lattice Theory, Amer. Math. Soc. Colloquium Publications 25 (New York, 1948).

2. Joe B. Rhodes, Modular and Distributive Semilattices, Ph.D. Thesis, University of Texas.

UNIVERSITY OF SOUTH CAROLINA

Columbia, South Carolina 